\title{
Ten-year results of tapered implants supporting single-tooth or fixed partial dentures
}

\author{
Stefano CORBELLA*, PhD, DDS, Laura DAL POZZO, DDS, Nicolò CAVALLI, PhD, DDS, Luca FRANCETTI, MD, DDS
}

Università degli Studi di Milano, Department of Biomedical, Surgical and Dental Sciences, Milan, Italy

IRCCS Istituto Ortopedico Galeazzi, Dental Clinic, Milan, Italy

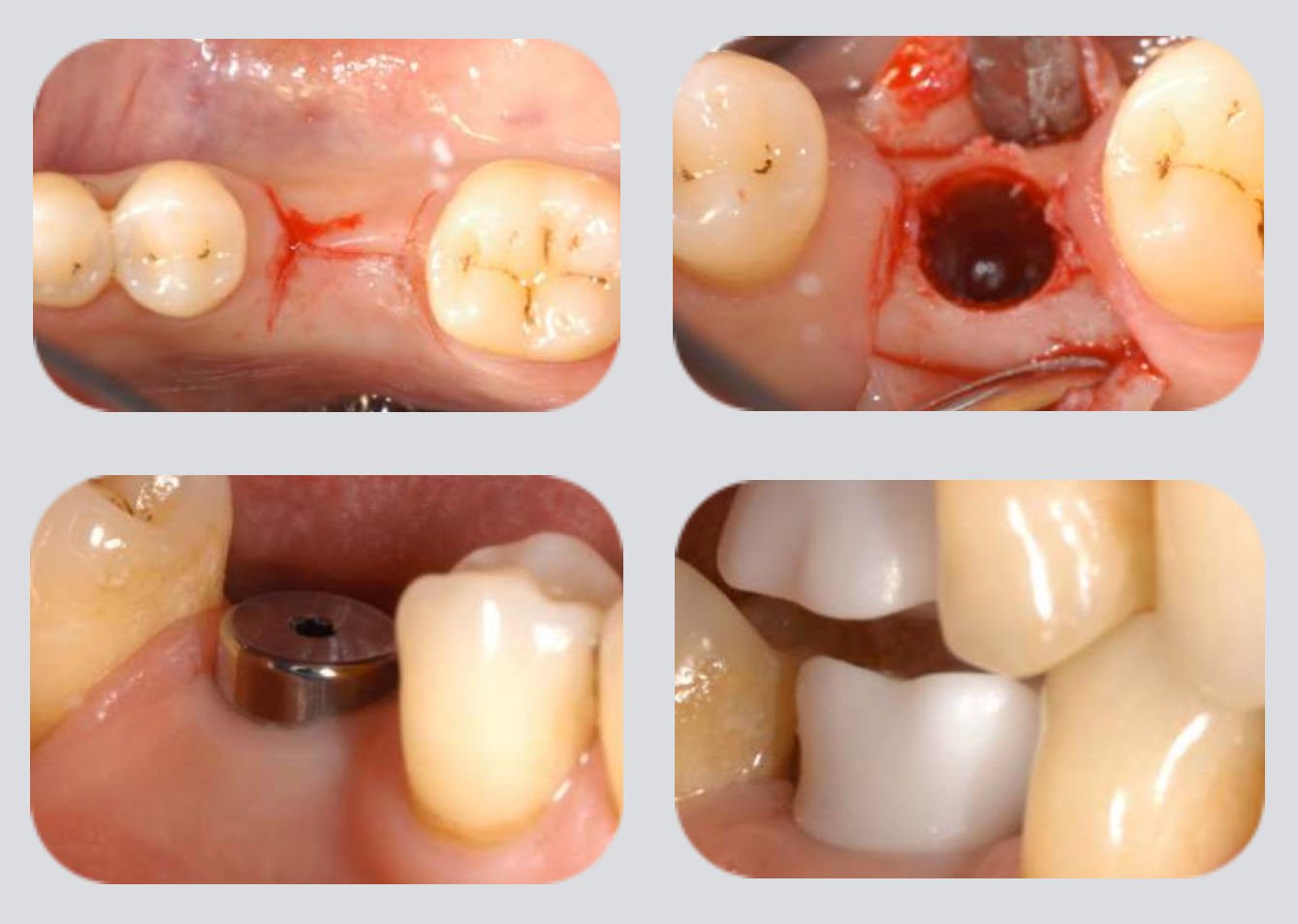

BACKGROUND AND AIM

Despite dental implants being widely used in clinical practice, there is a relative lack of information about medium- and long-term outcomes of implant-supported rehabilitations, particularly with respect to tapered implants and trends in bone resorption rates.

Among the parameters used to assess implant success, marginal bone resorption could be considered one of the most important, and also may influence implant survival ${ }^{1}$.

Marginal bone resorption can be classified as:

- early if it occurs during the first year of loading, which can be due to a number of factors (both biological and mechanical); or

- late, which may or may not follow an infective process, such as peri-implantitis ${ }^{1}$

The aim of this prospective single-center investigation was to evaluate the ten-year clinical outcomes, including marginal bone resorption, survival rate and prevalence of complications, of singletooth and partial rehabilitations supported by tapered implants.

\section{MATERIALS AND METHODS}

To be included in this single-center study, all subjects had to fulfill the following criteria: aged 18 years or more; classification as ASA-1 or ASA-2, an absence of any systemic condition that could influence marginal bone resorption ${ }^{2}$ (MBR); full-mouth plaque (FMPS) and bleeding (FMBS) scores less than $20 \%$; sufficient bone volume to place an implant without a bone regeneration procedure ${ }^{3}$.

All patients fulfilling the criteria were treated between June 2005 to March 2006 with tapered implants (Replace Select Tapered, Nobel Biocare AB, Göteborg, Sweden), using either a one- or two-stage protocol.

Bone quality ${ }^{4}$ was assessed at implant insertion on implant level.

Patients were recalled every six months for the first 3 years and then annually.

Mean MBR (mean of mesial/distal measurements from periapical radiographs, with prosthetic delivery as baseline), and occurrence of complications were recorded.

Unpaired student t-test was used to assess the significance of differences in MBRs.

Copyright @ 2017 Corbella S, Dal Pozzo L, Cavalli N, Francetti L

Contact: Dr. Stefano Corbella, PhD, DDS, stefano.corbella@gmail.com

\section{RESULTS}

A total of 19 patients ( 6 male, 13 female, mean age $64.60 \pm 8.59$ years) received 53 conical implants supporting 30 prosthetic rehabilitations comprising 13 single crown restorations and 17 fixed partial dentures (FPDs) and were followed for 10 years.

Prosthetic rehabilitation was done at $4.2 \pm 0.6$ months (range 3.0-4.8 months) after surgery.

Three implants failed due to peri-implantitis (one each after 2, 6 and 8 years from loading) and were removed, giving a cumulative survival rate of $95.4 \%$ and a cumulative success rate of $88.9 \%$ at 10 years after prosthetic loading.

The mean MBRs were $0.44 \pm 0.19 \mathrm{~mm}, 0.71 \pm 0.35 \mathrm{~mm}$, and $1.05 \pm 1.06 \mathbf{m m}$ after 1,5 and 10 years of loading, respectively. The mean MBR was statistically higher $(P=0.02)$ for implants placed in type 3 bone $(n=26)$ than for those placed in type 2 bone $(n=27)$. Furthermore, the mean MBR for implants supporting FPDs was statistically higher than for single crowns $(P=0.03)$.

\section{CONCLUSION AND CLINICAL IMPLICATIONS}

Tapered dental implants were characterized by a high cumulative survival and success rate, and low MBR changes at implant sites, up to 10 years after loading.

Given these medium-term outcomes, this study supports the clinical use of tapered implants for long-term FPDs and single-crown restorations.

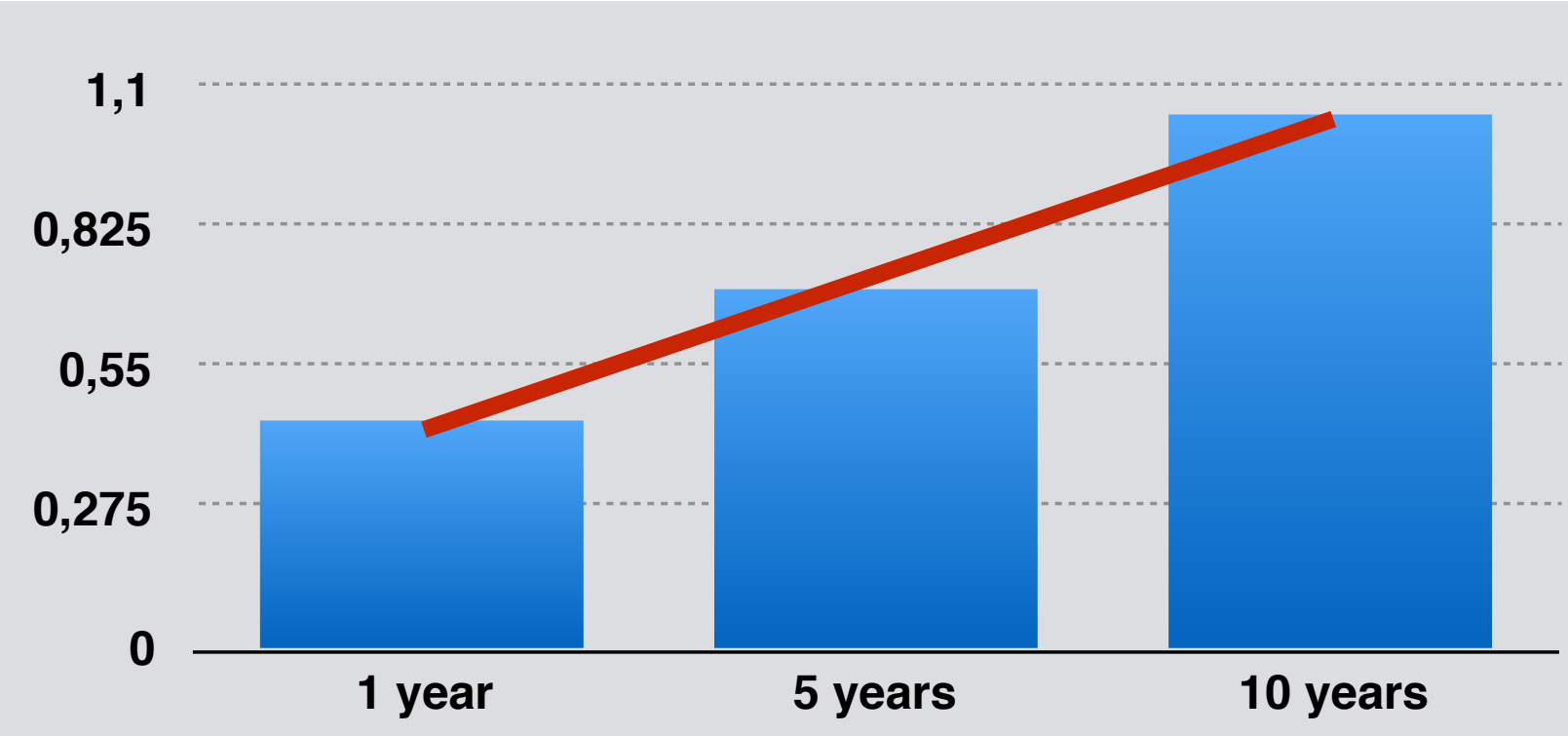

Figure 2.

Marginal bone resorption over time

2 Insua A, Monje A, Wang HL, Miron RJ. Basis of bone metabolism around dental implants during osseointegration and peri-implant bone loss. J Biomed Mater Res A 2017; 105(7): 2075-2089

${ }^{3}$ Ainamo J, Bay I. Problems and proposals for recording gingivitis and plaque. Int Dent J 1975; 25: 229-235 ${ }^{4}$ Lekholm U, Zarb GA. In: Patient selection and preparation. Tissue integrated prostheses: osseointegration ${ }^{4}$ Lekholm U, Zarb GA. In: Patient selection and preparation. Tissue integrated prostheses: osseointegration
in clinical dentistry. Branemark PI, Zarb GA, Albrektsson T, editor. Chicago: Quintessence Publishing

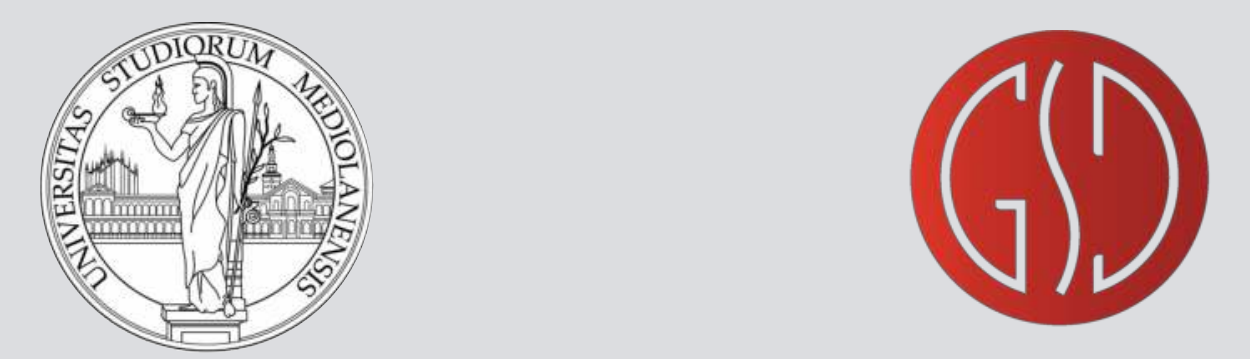

UNIVERSITÁ DEGI STUDI DI Mis.ANo 